\title{
Automatically Labeled Data Generation for Large Scale Event Extraction
}

\author{
Yubo Chen ${ }^{1,2}$, Shulin Liu ${ }^{1,2}$, Xiang Zhang ${ }^{1}$, Kang Liu ${ }^{1}$ and Jun Zhao ${ }^{1,2}$ \\ ${ }^{1}$ National Laboratory of Pattern Recognition, Institute of Automation, \\ Chinese Academy of Sciences, Beijing, 100190, China \\ ${ }^{2}$ University of Chinese Academy of Sciences, Beijing, 100049, China \\ \{yubo.chen, shulin.liu, xiang.zhang, kliu, jzhao\}@ nlpr.ia.ac.cn
}

\begin{abstract}
Modern models of event extraction for tasks like ACE are based on supervised learning of events from small hand-labeled data. However, hand-labeled training data is expensive to produce, in low coverage of event types, and limited in size, which makes supervised methods hard to extract large scale of events for knowledge base population. To solve the data labeling problem, we propose to automatically label training data for event extraction via world knowledge and linguistic knowledge, which can detect key arguments and trigger words for each event type and employ them to label events in texts automatically. The experimental results show that the quality of our large scale automatically labeled data is competitive with elaborately human-labeled data. And our automatically labeled data can incorporate with human-labeled data, then improve the performance of models learned from these data.
\end{abstract}

\section{Introduction}

Event Extraction (EE), a challenging task in Information Extraction, aims at detecting and typing events (Event Detection), and extracting arguments with different roles (Argument Identification) from natural-language texts. For example, in the sentence shown in Figure 1, an EE system is expected to identify an Attack event triggered by threw and extract the corresponding five augments with different roles: Yesterday (Role=Time $),$ demonstrators $($ Role $=$ Attacker $), s$ tones $($ Role=Instrument $)$, soldiers $($ Role $=$ Target $)$, and Israeli $($ Role $=$ Place $)$.

To this end, so far most methods (Nguyen et al.,

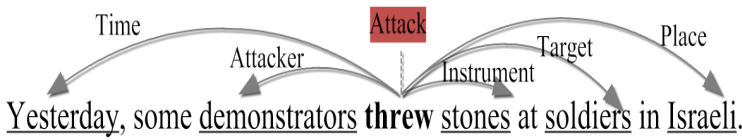

Figure 1: This sentence expresses an Attack event triggered by threw and containing five arguments.

2016; Chen et al., 2015; Li et al., 2014; Hong et al., 2011; Ji and Grishman, 2008) usually adopted supervised learning paradigm which relies on elaborate human-annotated data, such as ACE $2005^{1}$, to train extractors. Although this paradigm was widely studied, existing approaches still suffer from high costs for manually labeling training data and low coverage of predefined event types. In ACE 2005, all 33 event types are manually predefined and the corresponding event information (including triggers, event types, arguments and their roles) are manually annotated only in 599 English documents since the annotation process is extremely expensive. As Figure 2 shown, nearly $60 \%$ of event types in ACE 2005 have less than 100 labeled samples and there are even three event types which have less than ten labeled samples. Moreover, those predefined 33 event types are in low coverage for Natural Language Processing (NLP) applications on large-scale data.

Therefore, for extracting large scale events, especially in open domain scenarios, how to automatically and efficiently generate sufficient training data is an important problem. This paper aim$s$ to automatically generate training data for EE, which involves labeling triggers, event types, arguments and their roles. Figure 1 shows an example of labeled sentence. Recent improvements of Distant Supervision (DS) have been proven to be effective to label training data for Relation Extraction (RE), which aims to predict semantic re-

\footnotetext{
${ }^{1}$ http://projects.ldc.upenn.edu/ace/
} 


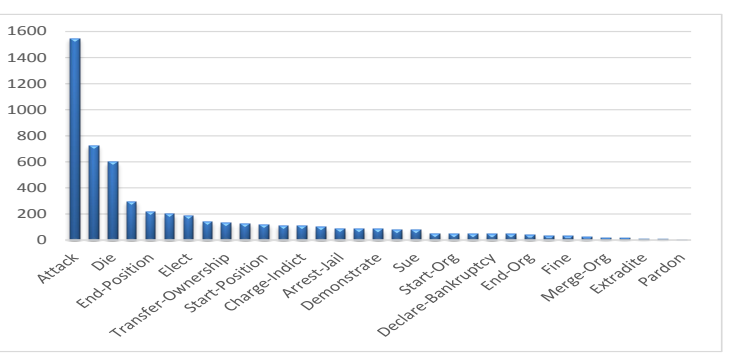

Figure 2: Statistics of ACE 2005 English Data.

lations between pairs of entities, formulated as (entity $y_{1}$, relation, entity $)_{2}$. And DS for RE assumes that if two entities have a relationship in a known knowledge base, then all sentences that mention these two entities will express that relationship in some way (Mintz et al., 2009). However, when we use DS for RE to EE, we meet following challenges:

Triggers are not given out in existing knowledge bases. EE aims to detect an event instance of a specific type and extract their arguments and roles, formulated as (event instance, event type; role $_{1}$, argument $_{1} ;$ role $_{2}$, argument $_{2} ; \ldots ;$ role $_{n}$, argument $_{n}$ ), which can be regarded as a kind of multiple or complicated relational data. In Figure 3, the right part shows an example of spouse_of relation between Barack Obama and Michelle Obama, where two rectangles represent two entities and the edge connecting them represents their relation. DS for RE uses two entities to automatically label training data; In comparison, the left part in Figure 3 shows a marriage event of Barack Obama and Michelle Obama, where the dash circle represents the marriage event instance of Barack Obama and Michelle Obama, rectangles represent arguments of the event instance, and each edge connecting an argument and the event instance expresses the role of the argument. For example, Barack Obama plays a Spouse role in this marriage event instance. It seems that we could use an event instance and an argument to automatically generate training data for argumen$t$ identification just like DS for RE. However, an event instance is a virtual node in existing knowledge bases and mentioned implicitly in texts. For example, in Freebase, the aforementioned marriage event instance is represented as $m .02 n q g l v$ (see details in Section 2). Thus we cannot directly use an event instance and an argument, like m.02nqglv and Barack Obama, to label back

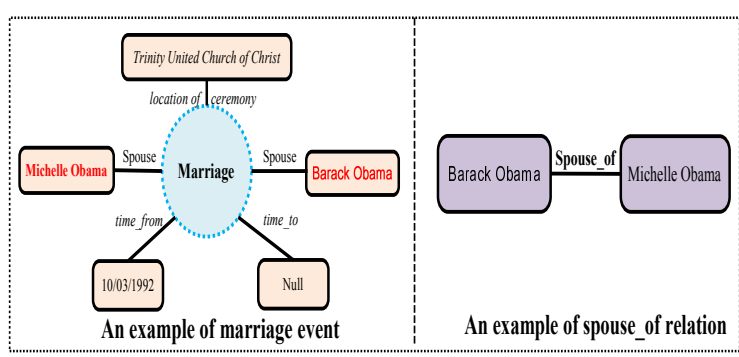

Figure 3: A comparison of events and relations.

in sentences. In $\mathrm{ACE}$ event extraction program, an event instance is represented as a trigger word, which is the main word that most clearly represents an event occurrence in sentences, like threw in Figure 1. Following ACE, we can use trigger words to represent event instance, like married for people.marriage event instance. Unfortunately, triggers are not given out in existing knowledge bases.

To resolve the trigger missing problem mentioned above, we need to discover trigger words before employing distant supervision to automatically label event arguments. Following DS in RE, we could naturally assume that a sentence contains all arguments of an event in the knowledge base tend to express that event, and the verbs occur in these sentences tend to evoke this type of events. However, arguments for a specific event instance are usually mentioned in multiple sentences. Simply employing all arguments in the knowledge base to label back in sentences will generate few sentences as training samples. As shown in Table 1, only $0.02 \%$ of instances can find all argument mentions in one sentence.

\begin{tabular}{|c|c|c|c|}
\hline Event Type & EI\# & A\# & S\# \\
\hline education.education & 530,538 & 8 & 0 \\
film.film_crew_gig & 252,948 & 3 & 8 \\
people.marriage & 152,276 & 5 & 0 \\
$\ldots$ & $\ldots$ & $\ldots$ & $\ldots$ \\
military.military_service & 27,933 & 6 & 0 \\
olympics.olympic_medal_honor & 20,790 & 5 & 4 \\
\hline sum of the selected 21 events & $3,870,492$ & 100 & 798 \\
\hline
\end{tabular}

Table 1: Statistics of events in Freebase. EI\# denotes number of event instances in Freebase. A\# denotes number of arguments for each event type$\mathrm{s}$, and $\mathrm{S \#}$ indicates number of sentences contain all arguments of each event type in Wikipedia.

To solve above problems, we propose an approach to automatically generate labeled data for large scale EE by jointly using world knowledge (Freebase) and linguistic knowledge (FrameNet). At first, we put forward an approach to prioritize 
arguments and select key or representative arguments (see details in Section 3.1) for each event type by using Freebase; Secondly, we merely use key arguments to label events and figure out trigger words; Thirdly, an external linguistic knowledge resource, FrameNet, is employed to filter noisy trigger words and expand more triggers; After that, we propose a Soft Distant Supervision (SDS) for EE to automatically label training data, which assumes that any sentence containing al1 key arguments in Freebase and a corresponding trigger word is likely to express that event in some way, and arguments occurring in that sentence are likely to play the corresponding roles in that event. Finally, we evaluate the quality of the automatically labeled training data by both manual and automatic evaluations. In addition, we employ a CNNbased EE approach with multi-instance learning for the automatically labeled data as a baseline for further research on this data. In summary, the contributions of this paper are as follows:

- To our knowledge, it is the first work to automatically label data for large scale EE via world knowledge and linguistic knowledge. All the labeled data in this paper have been released and can be downloaded freely ${ }^{2}$.

- We propose an approach to figure out key arguments of an event by using Freebase, and use them to automatically detect events and corresponding trigger words. Moreover, we employ FrameNet to filter noisy triggers and expand more triggers.

- The experimental results show that the quality of our large scale automatically labeled data is competitive with elaborately humanannotated data. Also, our automatically labeled data can augment traditional humanannotated data, which could significantly improve the extraction performance.

\section{Background}

In this paper, we respectively use Freebase as our world knowledge containing event instance and FrameNet as the linguistic knowledge containing trigger information. The articles in Wikipedia are used as unstructured texts to be labeled. To understand our method easily, we first introduce them as follows:

\footnotetext{
${ }^{2}$ https://github.com/acl2017submission/event-data
}

Freebase is a semantic knowledge base (Bollacker et al., 2008), which makes use of mediators (also called compound value types, CVTs) to merge multiple values into a single value. As shown in Figure 3, people.marriage is one type of CVTs. There are many instances of people.marriage and the marriage of Barack Obama and Michelle Obama is numbered as m.02nqglv. Spouse, from, to and location of ceremony are roles of the people.marriage CVTs. Barack Obama, Michelle Obama, 10/3/1992 and Trinity $U$ nited Church of Christ are the values of the instances. In this paper, we regard these CVTs as events, type of CVTs as event type, CVT instances as event instances, values in CVTs as arguments in events and roles of CVTs as the roles of arguments play in the event, respectively. According to the statistics of the Freebase released on $23^{\text {th }}$ April, 2015, there are around 1885 CVTs and around 14 million CVTs instances. After filtering out useless and meaningless CVTs, such as CVT$\mathrm{s}$ about user profiles and website information, we select 21 types of CVTs with around 3.8 million instances for experiments, which mainly involves events about education, military, sports and so on.

FrameNet $^{3}$ is a linguistic resource storing information about lexical and predicate argument semantics (Baker et al., 1998). FrameNet contains more than 1, 000 frames and 10,000 Lexical Units (LUs). Each frame of FrameNet can be taken as a semantic frame of a type of events (Liu et al., 2016). Each frame has a set of lemmas with part of speech tags that can evoke the frame, which are called LUs. For example, appoint.v is a LU of Appointing frame in FrameNet, which can be mapped to people.appointment events in Freebase. And a LUs of the frame plays a similar role as the trigger of an event. Thus we use FrameNet to detect triggers in our automatically data labeling process.

Wikipedia ${ }^{4}$ that we used was released on January, 2016. All 6.3 million articles in it are used in our experiments. We use Wikipedia because it is relatively up-to-date, and much of the information in Freebase is derived from Wikipedia.

\section{Method of Generating Training Data}

Figure 4 describes the architecture of automatically labeling data, which primarily involves the following four components: (i) Key argument de-

\footnotetext{
${ }^{3} \mathrm{http} / / /$ framenet.icsi.berkeley.edu

${ }^{4}$ https://www.wikipedia.org/
} 


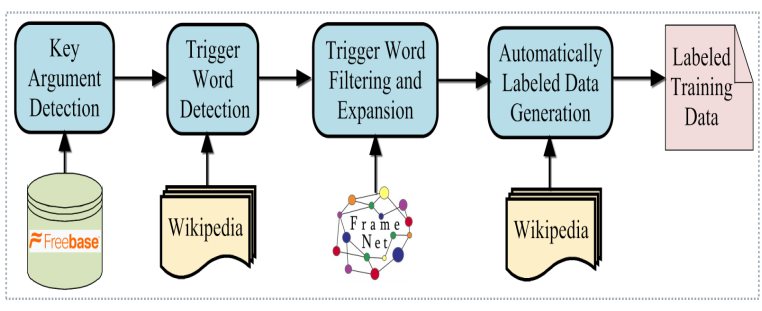

Figure 4: The architecture of automatically labeling training data for large scale event extraction.

tection, which prioritizes arguments of each event type and selects key arguments for each type of event; (ii) Trigger word detection, which uses key arguments to label sentences that may express events preliminarily, and then detect triggers; (iii) Trigger word filtering and expansion, which uses FrameNet to filter noisy triggers and expand triggers; (iv) Automatically labeled data generation, which uses a SDS to label events in sentences.

\subsection{Key Argument Detection}

This section illustrates how to detect key arguments for each event type via Freebase. Intuitively, arguments of a type of event play different roles. Some arguments play indispensable roles in an event, and serve as vital clues when distinguishing different events. For example, compared with arguments like time, location and so on, spouses are key arguments in a marriage event. We call these arguments as key arguments. We propose to use Key Rate (KR) to estimate the importance of an argument to a type of event, which is decided by two factors: Role Saliency and Event Relevance.

Role Saliency (RS) reflects the saliency of an argument to represent a specific event instance of a given event type. If we tend to use an argumen$t$ to distinguish one event instance form other instances of a given event type, this argument will play a salient role in the given event type. We define RS as follows:

$$
R S_{i j}=\frac{\operatorname{Count}\left(A_{i}, E T_{j}\right)}{\operatorname{Count}\left(E T_{j}\right)}
$$

where $R S_{i j}$ is the role saliency of $i$-th argument to $j$-th event type, $\operatorname{Count}\left(A_{i}, E T_{j}\right)$ is the number of Arguemnt $t_{i}$ occurring in all instances of eventType $_{j}$ in Freebase and Count $\left(E T_{j}\right)$ is the number of instances of eventType ${ }_{j}$ in Freebase.

Event Relevance (ER) reflects the ability in which an argument can be used to discriminate d- ifferent event types. If an argument occurs in every event type, the argument will have a low event relevance. We propose to compute ER as follows:

$$
E R_{i}=\log \frac{\operatorname{Sum}(E T)}{1+\operatorname{Count}(E T C i)}
$$

where $E R_{i}$ is the event relevance of $i$-th argument, Sum $(E T)$ is the number of all event types in knowledge base and $\operatorname{Count}\left(E T C_{i}\right)$ is the number of event types containing $i$-th argument. Finally, $\mathrm{KR}$ is computed as follows:

$$
K R_{i j}=R S_{i j} * E R_{i}
$$

We compute KR for all arguments of each even$\mathrm{t}$ type, and sort them according to KR. Then we choose top $K$ arguments as key arguments.

\subsection{Trigger Word Detection}

After detecting key arguments for every even$t$ types, we use these key arguments to label sentences that may express events in Wikipedia. At first, we use Standford CoreNLP tool ${ }^{5}$ to converts the raw Wikipedia texts into a sequence of sentences, attaches NLP annotations (POS tag, NER tag). Finally, we select sentences that contains all key arguments of an event instance in Freebase as sentences expressing corresponding events. Then we use these labeled sentences to detect triggers.

In a sentence, a verb tend to express an occurrence of an event. For example, in ACE 2005 English data, there are $60 \%$ of events triggered by verbs. As shown in Figure 1, threw is a trigger of Attack event. Intuitively, if a verb occurs more times than other verbs in the labeled sentences of one event type, the verb tends to trigger this type of event; and if a verb occurs in sentences of every event types, like is, the verb will have a low probability to trigger events. Thus we propose Trigger Candidate Frequency (TCF) and Trigger Event Type Frequency (TETF) to evaluate above two aspects. Finally we employ Trigger Rate (TR), which is the product of TCF and TETF to estimate the probability of a verb to be a trigger, which is formulated as follows:

$$
\begin{gathered}
T R_{i j}=T C F_{i j} * T E T F_{i} \\
T C F_{i j}=\frac{\operatorname{Count}\left(V_{i}, E T S_{j}\right)}{\operatorname{Count}\left(E T S_{j}\right)}
\end{gathered}
$$

\footnotetext{
${ }^{5} \mathrm{http} / / /$ stanfordnlp.github.io/CoreNLP/
} 


$$
T E T F_{i}=\log \frac{\operatorname{Sum}(E T)}{1+\operatorname{Count}\left(E T I_{i}\right)}
$$

where $T R_{i j}$ is the trigger rate of $i$-th verb to $j$ th event type, Count $\left(V_{i}, E T S_{j}\right)$ is the number of sentences, which express $j$-th type of event and contain $i$-th verb, $\operatorname{Count}\left(E T S_{j}\right)$ is the number of sentences expressing $j$-th event type, $\operatorname{Count}\left(E T I_{i}\right)$ is the number of event types, which have the labeled sentences containing $i$-th verb. Finally, we choose verbs with high $T R$ values as the trigger words for each event type.

\subsection{Trigger Word Filtering and Expansion}

We can obtain an initial verbal trigger lexicon by above trigger word detection. However, this initial trigger lexicon is noisy and merely contains verbal triggers. The nominal triggers like marriage are missing. Because the number of nouns in one sentence is usually larger than that of verbs, it is hard to use TR to find nominal triggers. Thus, we propose to use linguistic resource FrameNet to filter noisy verbal triggers and expand nominal triggers. As the success of word embedding in capturing semantics of words (Turian et al., 2010), we employ word embedding to map the events in Freebase to frames in FrameNet. Specifically, we use the average word embedding of all words in $i$-th Freebase event type name $e_{i}$ and word embedding of $k$-th lexical units of $j$-th frame $e_{j, k}$ to compute the semantic similarity. Finally, we select the frame contains max similarity of $e_{i}$ and $e_{j, k}$ as the mapped frame, which can be formulated as follows:

$$
\text { frame }(i)=\arg \max _{j}\left(\operatorname{similarity}\left(e_{i}, e_{j, k}\right)\right)
$$

Then, we filter the verb, which is in initial verbal trigger word lexicon and not in the mapping frame. And we use nouns with high confidence in the mapped frame to expand trigger lexicon.

\subsection{Automatically labeled data generation}

Finally, we propose a Soft Distant Supervision and use it to automatically generate training data, which assumes that any sentence containing al1 key arguments in Freebase and a corresponding trigger word is likely to express that event in some way, and arguments occurring in that sentence are likely to play the corresponding roles in that event.

\section{Method of Event Extraction}

In this paper, event extraction is formulated as a two-stage, multi-class classification task. The first stage is called Event Classification, which aims to predict whether the key argument candidates participate in a Freebase event. If the key arguments participate a Freebase event, the second stage is conducted, which aims to assign arguments to the event and identify their corresponding roles. We call this stage as argumen$t$ classification. We employ two similar Dynamic Multi-pooling Convolutional Neural Networks with Multi-instance Learning (DMCNNs-MIL) for above two stages. The Dynamic Multi-pooling Convolutional Neural Networks (DMCNNs) is the best reported CNN-based model for event extraction (Chen et al., 2015) by using human-annotated training data. However, our automatically labeled data face a noise problem, which is a intrinsic problem of using DS to construct training data (Hoffmann et al., 2011; Surdeanu et al., 2012). In order to alleviate the wrong label problem, we use Multi-instance Learning (MIL) for two DMCNNs. Because the second stage is more complicated and limited in space, we take the MIL used in arguments classification as an example and describes as follows:

We define all of the parameters for the stage of argument classification to be trained in DMCNNs as $\theta$. Suppose that there are $T$ bags $\left\{M_{1}, M_{2}, \ldots, M_{T}\right\}$ and that the $i$-th bag contains $q_{i}$ instances (sentences) $M_{i}=\left\{m_{i}^{1}, m_{i}^{2}, \ldots, m_{i}^{q_{i}}\right\}$, the objective of multi-instance learning is to predict the labels of the unseen bags. In stage of argument classification, we take sentences containing the same argument candidate and triggers with a same event type as a bag and all instances in a bag are considered independently. Given an input instance $m_{i}^{j}$, the network with the parameter $\theta$ outputs a vector $O$, where the $r$-th component $O_{r}$ corresponds to the score associated with argument role $r$. To obtain the conditional probability $p\left(r \mid m_{i}^{j}, \theta\right)$, we apply a softmax operation over all argument role types:

$$
p\left(r \mid m_{i}^{j}, \theta\right)=\frac{e^{o_{r}}}{\sum_{k=1}^{n} e^{o_{k}}}
$$

where, $n$ is the number of roles. And the objective of multi-instance learning is to discriminate bags rather than instances. Thus, we define the objective function on the bags. Given all $(T)$ training bags $\left(M_{i}, y_{i}\right)$, we can define the objective function 


\begin{tabular}{|c|c|c|c|c|c|}
\hline Event Type & $\begin{array}{l}\text { Freebase } \\
\text { Size }\end{array}$ & $\begin{array}{l}\text { Sentences } \\
(\mathrm{KA})\end{array}$ & $\begin{array}{c}\text { Sentences } \\
(\mathrm{KA}+\mathrm{T})\end{array}$ & Examples of argument roles sorted by KR & Examples of triggers \\
\hline people.marriage & 152,276 & 56,837 & 26,349 & spouse, spouse, from, to, location & marriage, marry, wed, wedding, couple,..., wife \\
\hline music.group_membership & 239,813 & 90,617 & 20,742 & group, member, start, role, end & musician, singer, sing, sang, sung, concert,..., play \\
\hline education.education & 530,538 & 26,966 & 11,849 & student, institution, degree,..., minor & educate, education, graduate, learn, study,..., student \\
\hline organization.leadership & 43,610 & 5,429 & 3,416 & organization, person, title,..., to & CEO, charge, administer, govern, rule, boss,..., chair \\
\hline olympics.olympic_medal_honor & 20,790 & 4,056 & 2,605 & medalist, olympics, event,..., country & win, winner, tie, victor, gold, silver,..., bronze \\
\hline$\ldots$ & $\ldots$ & $\ldots$ & $\ldots$ & $\ldots$ & . \\
\hline sum of 21 selected events & $3,870,492$ & 421,602 & 72,611 & argument 1 , argument $2, \ldots$, argumentN & trigger1, trigger2, trigger $3, \ldots$, triggerN \\
\hline
\end{tabular}

Table 2: The statistics of five largest automatically labeled events in selected 21 Freebase events, with their size of instances in Freebase, sentences labeled with key argument (KA) and KA + Triggers(T), examples of arguments roles sorted by KR and examples of triggers.

using cross-entropy at the bag level as follows:

$$
J(\theta)=\sum_{i=1}^{T} \log p\left(y_{i} \mid m_{i}^{j}, \theta\right)
$$

where $j$ is constrained as follows:

$$
j^{*}=\arg \max _{j} p\left(r \mid m_{i}^{j}, \theta\right) \quad 1 \leq j \leq q_{i}
$$

To compute the network parameter $\theta$, we maximize the $\log$ likelihood $J(\theta)$ through stochastic gradient descent over mini-batches with the Adadelta (Zeiler, 2012) update rule.

\section{Experiments}

In this section, we first manually evaluate our automatically labeled data. Then, we conduct automatic evaluations for our labeled data based on ACE corpus and analyze effects of different approaches to automatically label training data. Finally, we shows the performance of DMCNNs-MIL on our automatically labeled data.

\subsection{Our Automatically Labeled Data}

By using the proposed methods, a large set of labeled data could be generated automatically. Table 2 shows the statistics of the five largest automatically labeled events among selected 21 Freebase events. Two hyper parameters, the number of key arguments and the value of TR in our automatically data labeling, are set as 2 and 0.8 , by grid search respectively. When we merely use two key arguments to label data, we will obtain 421, 602 labeled sentences. However, these sentences miss labeling triggers. Thus, we leverage these rough labeled data and FrameNet to find triggers and use SDS to generate labeled data. Finally, 72,611 labeled sentences are generated automatically. Compared with nearly 6,000 human annotated labeled sentence in ACE, our method can automatically generate large scale labeled training data.

\subsection{Manual Evaluations of Labeled Data}

\author{
\#\#01 He is the uncle of [Amal Clooney], [wife] of the actor [George Clooney]. \\ Trigger: wife Event Type: Marriage MannalAnotate[Y/N]: \\ Argument: Amal Clooney Role:Spouse MannalAnotate[Y/N] \\ Argument: George Clooney Role:Spouse MannalAnotate[Y/N]:

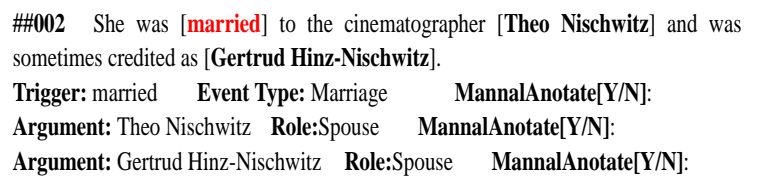

Figure 5: Examples of manual evaluations.

We firstly manually evaluate the precision of our automatically generated labeled data. We randomly select 500 samples from our automatically labeled data. Each selected sample is a sentence with a highlighted trigger, labeled arguments and corresponding event type and argument roles. Figure 5 gives some samples. Annotators are asked to assign one of two labels to each sample. "Y": the word highlighted in the given sentence indeed triggers an event of the corresponding type or the word indeed plays the corresponding role in that event. Otherwise " $\mathrm{N}$ " is labeled. It is very easy to annotate a sample for annotators, thus the annotated results are expected to be of high quality. Each sample is independently annotated by three annotators $^{6}$ (including one of the authors and two of our colleagues who are familiar with event extraction task) and the final decision is made by voting.

\begin{tabular}{|c|c|}
\hline Stage & Average Precision \\
\hline Trigger Labeling & 88.9 \\
\hline Argument Labeling & 85.4 \\
\hline
\end{tabular}

Table 3: Manual Evaluation Results

We repeat above evaluation process on the final 72,611 labeled data three times and the average precision is shown in Table 3. Our automatically generated data can achieve a precision of 88.9 and 85.4 for trigger labeling and argument labeling re-

\footnotetext{
${ }^{6}$ The inter-agreement rate is $87.5 \%$
} 


\begin{tabular}{|c|c|c|c|c|c|c|c|c|c|c|c|c|}
\hline \multirow[t]{2}{*}{ Methods } & \multicolumn{3}{|c|}{$\begin{array}{c}\text { Trigger } \\
\text { Identification(\%) }\end{array}$} & \multicolumn{3}{|c|}{$\begin{array}{l}\text { Trigger Identification } \\
+ \text { Classification }(\%)\end{array}$} & \multicolumn{3}{|c|}{$\begin{array}{c}\text { Argument } \\
\text { Identification(\%) }\end{array}$} & \multicolumn{3}{|c|}{$\begin{array}{l}\text { Argument } \\
\operatorname{Role}(\%)\end{array}$} \\
\hline & $\mathrm{P}$ & $\mathrm{R}$ & $\mathrm{F}$ & $\mathrm{P}$ & $\mathrm{R}$ & $\mathrm{F}$ & & $\mathrm{R}$ & $\mathrm{F}$ & & $\mathrm{R}$ & $\mathrm{F}$ \\
\hline cture trained $\mathrm{w}$ & 76.9 & 65.0 & 70.4 & 73.7 & 62.3 & 67.5 & 69.8 & 47.9 & 56.8 & 64.7 & 44.4 & 52.7 \\
\hline Chen & 80.4 & 67.7 & 73.5 & 75.6 & 63.6 & 69.1 & 68.8 & 51.9 & 59.1 & 62.2 & 46.9 & 53.5 \\
\hline trained with ACE & 68.5 & 75.7 & 71.9 & 66.0 & 73.0 & 69.3 & 61.4 & 64.2 & 62.8 & 54.2 & 56.7 & 55.4 \\
\hline$\overline{\mathrm{DMCl}}$ & 77.6 & 67.7 & 72.3 & 72.9 & 63.7 & 68.0 & 64.9 & 51.7 & 57.6 & 58.7 & 46.7 & 52.0 \\
\hline DMCNN trained with ACE+ED & 79.7 & 69.6 & 74.3 & 75.7 & 66.0 & 70.5 & 71.4 & 56.9 & 63.3 & 62.8 & 50.1 & 55.7 \\
\hline
\end{tabular}

Table 4: Overall performance on ACE blind test data

spectively, which demonstrates that our automatically labeled data is of high quality.

\subsection{Automatic Evaluations of Labeled Data}

To prove the effectiveness of the proposed approach automatically, we add automatically generated labeled data into ACE dataset to expand the training sets and see whether the performance of the event extractor trained on such expanded training sets is improved. In our automatically labeled data, there are some event types that can correspond to those in ACE dataset. For example, our people.marriage events can be mapped to life.marry events in ACE2005 dataset. We mapped these types of events manually and we add them into ACE training corpus in two ways. (1) we delete the human annotated ACE data for these mapped event types in ACE dataset and add our automatically labeled data to remainder ACE training data. We call this Expanded Data (ED) as ED Only. (2) We directly add our automatically labeled data of mapped event types to ACE training data and we call this training data as $A C E+E D$. Then we use such data to train the same even$\mathrm{t}$ extraction model (DMCNN) and evaluate them on the ACE testing data set. Following (Nguyen et al., 2016; Chen et al., 2015; Li et al., 2013), we used the same test set with 40 newswire articles and the same development set with 30 documents and the rest 529 documents are used for ACE training set. And we use the same evaluation metric $P$, $\mathrm{R}, \mathrm{F}$ as ACE task defined. We select three baselines trained with ACE data. (1) Li's structure, which is the best reported structured-based system (Li et al., 2013). (2) Chen's DMCNN, which is the best reported CNN-based system (Chen et al., 2015). (3) Nguyen's JRNN, which is the state-ofthe-arts system (Nguyen et al., 2016).

The results are shown in Table 4. Compared with all models, DMCNN trained with $A C E+E D$ achieves the highest performance. This demonstrates that our automatically generated labeled data could expand human annotated training data effectively. Moreover, compared with Chen's DMCNN trained with ACE, DMCNN trained with ED Only achieves a competitive performance. This demonstrates that our large scale automatically labeled data is competitive with elaborately humanannotated data.

\subsection{Discussion}

\section{Impact of Key Rate}

In this section, we prove the effectiveness of KR to find key arguments and explore the impact of different numbers of key arguments to automatically generate data. We specifically select two methods as baselines for comparison with our KR method: ER and RS, which use the event relevance and role salience to sort arguments of each type of events respectively. Then we choose the same number of key arguments in all methods and use these key arguments to label data. After that we evaluate these methods by using above automatic evaluations based on ACE data. Results are shown in Table 5. $A C E+K R$ achieve the best performance in both stages. This demonstrates the effectiveness of our KR methods.

\begin{tabular}{|c|c|c|}
\hline \multirow{2}{*}{ Feature } & Trigger & Argument \\
\cline { 2 - 3 } & $F_{1}$ & $F_{1}$ \\
\hline ACE & 69.1 & 53.5 \\
\hline ACE + RS & 70.1 & 55.3 \\
\hline ACE + ER & 69.5 & 54.2 \\
\hline ACE + KR & $\mathbf{7 0 . 5}$ & $\mathbf{5 5 . 7}$ \\
\hline
\end{tabular}

Table 5: Effects of ER, RS and KR

To explore the impact of different numbers of key arguments, we sort all arguments of each type of events according to KR value and select top $k$ arguments as the key arguments. Examples are shown in Table 2. Then we automatically evaluate the performance by using automatic evaluations proposed above. Figure 6 shows the results, when we set $k=2$, the method achieves a best 


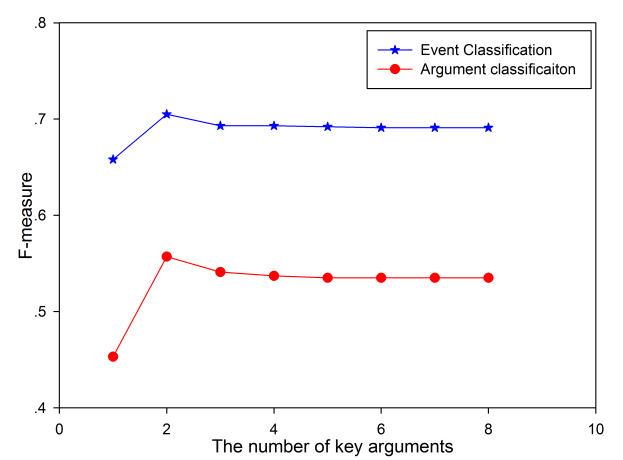

Figure 6: Effects of the number of key arguments

performance in both stages. Then, the F1 value reduces as $k$ grows. The reason is that the heuristics for data labeling are stricter as $k$ grows. As a result, less training data is generated. For example, if $k=2$, we will get 25,797 sentences labeled as people.marriage events and we will get 534 labeled sentences, if $k=3$. However, when we set $k=1$, although more labeled data are generated, the precision could not be guaranteed.

\section{Impact of Trigger Rate and FrameNet}

In this section, we prove the effectiveness of TR and FrameNet to find triggers. We specifically select two methods as baselines: TCF and TETF. TCF, TETF and TR respectively use the trigger candidate frequency, trigger event type frequency and trigger rate to sort trigger candidates of each type of events. Then we generate initial trigger lexicon by using all trigger candidates with high TCF value, TETF value or TR value. We set these hyper parameters as $0.8,0.9$ and 0.8 , respectively, which are determined by grid search from $(0.5,0.6,0.7,0.8,0.9,1.0)$. FrameNet was used to filter noisy verbal triggers and expand nominal triggers. Trigger examples generated by $T R+$ Framenet are shown in Table 2. Then we evaluate the performance of these methods by using above automatic evaluations. Results are shown in Table 6, Compared with $A C E+T C F$ and $A C E+T E T F, A C E+T R$ gains a higher performance in both stages. It demonstrates the effectiveness of our TR methods. When we use FrameNet to generate triggers, compared with $A C E+T R$, we get a 1.0 improvement on trigger classification and a 1.7 improvement on argument classification. Such improvements are higher than improvements gained by other methods (TCF, IEF, TR), which demonstrates the effectiveness of the usage of FrameNet.

\begin{tabular}{|c|c|c|}
\hline \multirow{2}{*}{ Feature } & Trigger & Argument \\
\cline { 2 - 3 } & $F_{1}$ & $F_{1}$ \\
\hline ACE & 69.1 & 53.5 \\
\hline ACE + TCF & 69.3 & 53.8 \\
\hline ACE + TETF & 69.2 & 53.7 \\
\hline ACE + TR & 69.5 & 54.0 \\
\hline ACE + TR + FrameNet & $\mathbf{7 0 . 5}$ & $\mathbf{5 5 . 7}$ \\
\hline
\end{tabular}

Table 6: Effects of TCF, TETF,TR and FrameNet

\subsection{Performance of DMCNN-MIL}

Following previous work (Mintz et al., 2009) in distant supervised RE, we evaluate our method in two ways: held-out and manual evaluation.

\section{Held-out Evaluation}

In the held-out evaluation, we hold out part of the Freebase event data during training, and compare newly discovered event instances against this heldout data. We use the following criteria to judge the correctness of each predicted event automatically: (1) An event is correct if its key arguments and event type match those of an event instance in Freebase; (2) An argument is correctly classified if its event type and argument role match those of any of the argument instance in the corresponding Freebase event. Figure 7 and Figure 8 show the precision-recall (P-R) curves for each method in the two stages of event extraction respectively. We can see that multi-instance learning is effective to alleviate the noise problem in our distant supervised event extraction.
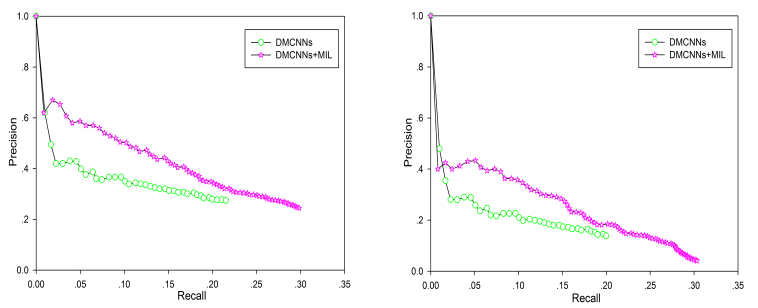

Figure 7: P-R curves for Figure 8: P-R curves for event classification. argument classification.

\section{Human Evaluation}

Because the incomplete nature of Freebase, heldout evaluation suffers from false negatives problem. We also perform a manual evaluation to eliminate these problems. In the manual evaluation, we manually check the newly discovered event instances that are not in Freebase. Because the number of these event instances in the test data is unknown, we cannot calculate the recall in this case. 
Instead, we calculate the precision of the top $\mathrm{n}$ extracted event instances. The human evaluation results are presented in Table 7 . We can see that DMCNNs-MIL achieves the best performance.

\begin{tabular}{|c|c|c|c|c|}
\hline \multirow{2}{*}{ Methods } & \multicolumn{4}{|c|}{ Event Classificaiton } \\
\cline { 2 - 5 } & Top 100 & Top 300 & Top 500 & Average \\
\hline DMCNNs & 58.7 & 54.3 & 52.9 & 55.3 \\
\hline DMCNNs+MIL & $\mathbf{7 0 . 6}$ & $\mathbf{6 7 . 2}$ & $\mathbf{6 4 . 3}$ & $\mathbf{6 7 . 4}$ \\
\hline \hline \multirow{2}{*}{ Methods } & \multicolumn{4}{|c|}{ Argument Classificaiton } \\
\cline { 2 - 5 } & Top 100 & Top 300 & Top 500 & Average \\
\hline DMCNNs & 43.5 & 40.6 & 36.7 & 40.3 \\
\hline DMCNNs+MIL & $\mathbf{5 0 . 8}$ & $\mathbf{4 5 . 6}$ & $\mathbf{4 3 . 5}$ & $\mathbf{4 6 . 6}$ \\
\hline
\end{tabular}

Table 7: Precision for top 100, 300, and 500 events

\section{Related Work}

Most of previous event extraction work focused on supervised learning paradigm and trained event extractors on human-annotated data which yield relatively high performance. (Ahn, 2006; Ji and Grishman, 2008; Hong et al., 2011; McClosky et al., 2011; Li et al., 2013, 2014; Chen et al., 2015; Nguyen and Grishman, 2015; Nguyen et al., 2016). However, these supervised methods depend on the quality of the training data and labeled training data is expensive to produce. Unsupervised methods can extract large numbers of events without using labeled data (Chambers and Jurafsky, 2011; Cheung et al., 2013; Huang et al., 2016). But extracted events may not be easy to be mapped to events for a particular knowledge base.

Distant supervision have been used in relation extraction for automatically labeling training data (Mintz et al., 2009; Hinton et al., 2012; Krause et al., 2012; Krishnamurthy and Mitchell, 2012; Berant et al., 2013; Surdeanu et al., 2012; Zeng et al., 2015). But DS for RE cannot directly use for EE. For the reasons that an event is more complicated than a relation and the task of EE is more difficult than RE. The best reported supervised RE and EE system got a F1-score of $88.0 \%$ (Wang et al., 2016) and $55.4 \%$ (Nguyen et al., 2016) respectively. Reschke et al. (2014) extended the distant supervision approach to fill slots in plane crash. However, the method can only extract arguments of one plane crash type and need flight number strings as input. In other words, the approach cannot extract whole event with different types automatically.

\section{Conclusion and Future Work}

In this paper, we present an approach to automatically label training data for EE. The experimental results show the quality of our large scale automatically labeled data is competitive with elaborately human-annotated data. Also, we provide a DMCNN-MIL model for this data as a baseline for further research. In the future, we will use the proposed automatically data labeling method to more event types and explore more models to extract events by using automatically labeled data.

\section{Acknowledgments}

This work was supported by the Natural Science Foundation of China (No. 61533018) and the National Basic Research Program of China (No. 2014CB340503). And this research work was also supported by Google through focused research awards program.

\section{References}

David Ahn. 2006. The stages of event extraction. In Proceedings of the Workshop on Annotating and Reasoning About Time and Events. pages 1-8. $\mathrm{http}: / / \mathrm{dl} . \mathrm{acm}$. org/citation.cfm?id=1629235.1629236.

Collin F Baker, Charles J Fillmore, and John B Lowe. 1998. The berkeley framenet project. In Proceedings of the 36th Annual Meeting of the Association for Computational Linguistics and 17th International Conference on Computational Linguistics. Association for Computational Linguistics, pages 86-90. http://aclweb.org/anthology/C98-1013.

Jonathan Berant, Andrew Chou, Roy Frostig, and Percy Liang. 2013. Semantic parsing on freebase from question-answer pairs. In Proceedings of the 2013 Conference on Empirical Methods in Natural Language Processing. Association for Computational Linguistics, pages 1533-1544. http://aclweb.org/anthology/D13-1160.

Kurt Bollacker, Colin Evans, Praveen Paritosh, Tim Sturge, and Jamie Taylor. 2008. Freebase: a collaboratively created graph database for structuring human knowledge. In Proceedings of the 2008 ACM SIGMOD international conference on Management of data. pages 1247-1250. http://doi.acm.org/10.1145/1376616.1376746.

Nathanael Chambers and Dan Jurafsky. 2011. Template-based information extraction without the templates. In Proceedings of the 49th Annual Meeting of the Association for Computational Linguistics: Human Language Technologies. Association for Computational Linguistics, pages 976-986. http://aclweb.org/anthology/P11-1098.

Yubo Chen, Liheng Xu, Kang Liu, Daojian Zeng, and Jun Zhao. 2015. Event extraction via dynamic multi-pooling convolutional neural networks. In Proceedings of the 53rd Annual Meet- 
ing of the Association for Computational Linguistics and the 7th International Joint Conference on Natural Language Processing. Association for Computational Linguistics, pages 167-176. https://doi.org/10.3115/v1/P15-1017.

Kit Jackie Chi Cheung, Hoifung Poon, and Lucy Vanderwende. 2013. Probabilistic frame induction. In Proceedings of the 2013 Conference of the North American Chapter of the Association for Computational Linguistics: Human Language Technologies. Association for Computational Linguistics, pages 837-846. http://aclweb.org/anthology/N13-1104.

Geoffrey E Hinton, Nitish Srivastava, Alex Krizhevsky, Ilya Sutskever, and Ruslan R Salakhutdinov. 2012. Improving neural networks by preventing coadaptation of feature detectors. arXiv preprint arXiv:1207.0580 https://arxiv.org/pdf/1207.0580.

Raphael Hoffmann, Congle Zhang, Xiao Ling, Luke Zettlemoyer, and S. Daniel Weld. 2011. Knowledge-based weak supervision for information extraction of overlapping relations. In Proceedings of the 49th Annual Meeting of the Association for Computational Linguistics: Human Language Technologies. Association for Computational Linguistics, pages 541-550. http://aclweb.org/anthology/P111055 .

Yu Hong, Jianfeng Zhang, Bin Ma, Jianmin Yao, Guodong Zhou, and Qiaoming Zhu. 2011. Using cross-entity inference to improve event extraction. In Proceedings of the 49th Annual Meeting of the Association for Computational Linguistics: Human Language Technologies. Association for Computational Linguistics, pages 1127-1136. http://aclweb.org/anthology/P11-1113.

Lifu Huang, Taylor Cassidy, Xiaocheng Feng, Heng Ji, Clare R. Voss, Jiawei Han, and Avirup Sil. 2016. Liberal event extraction and event schema induction. In Proceedings of the 54th Annual Meeting of the Association for Computational Linguistics. Association for Computational Linguistics, pages 258-268. http://www.aclweb.org/anthology/P16-1025.

Heng Ji and Ralph Grishman. 2008. Refining event extraction through cross-document inference. In Proceedings of ACL-08: HLT. Association for Computational Linguistics, pages 254-262. http://aclweb.org/anthology/P08-1030.

Sebastian Krause, Hong Li, Hans Uszkoreit, and Feiyu Xu. 2012. Large-scale learning of relationextraction rules with distant supervision from the web. In Proceedings of International Semantic Web Conference, Springer, pages 263278. http://link.springer.com/chapter/10.1007/9783-642-35176-1_17.

Jayant Krishnamurthy and Tom Mitchell. 2012. Weakly supervised training of semantic parsers. In Proceedings of the 2012 Joint Conference on Empirical Methods in Natural Language Processing and
Computational Natural Language Learning. Association for Computational Linguistics, pages 754-765. http://aclweb.org/anthology/D12-1069.

Qi Li, Heng Ji, Yu Hong, and Sujian Li. 2014. Constructing information networks using one single model. In Proceedings of the 2014 Conference on Empirical Methods in Natural Language Processing. Association for Computational Linguistics, pages 1846-1851. https://doi.org/10.3115/v1/D141198.

Qi Li, Heng Ji, and Liang Huang. 2013. Joint event extraction via structured prediction with global features. In Proceedings of the 51st Annual Meeting of the Association for Computational Linguistics. Association for Computational Linguistics, pages 7382. http://aclweb.org/anthology/P13-1008.

Shulin Liu, Yubo Chen, Shizhu He, Kang Liu, and Jun Zhao. 2016. Leveraging framenet to improve automatic event detection. In Proceedings of the 54th Annual Meeting of the Association for Computational Linguistics. Association for Computational Linguistics, pages 2134-2143. http://www.aclweb.org/anthology/P16-1201.

David McClosky, Mihai Surdeanu, and Christopher Manning. 2011. Event extraction as dependency parsing. In Proceedings of the 49th Annual Meeting of the Association for Computational Linguistics: Human Language Technologies. Association for Computational Linguistics, pages 1626-1635. http://aclweb.org/anthology/P11-1163.

Mike Mintz, Steven Bills, Rion Snow, and Daniel Jurafsky. 2009. Distant supervision for relation extraction without labeled data. In Proceedings of the Joint Conference of the 47th Annual Meeting of the $A C L$ and the 4th International Joint Conference on Natural Language Processing of the AFNLP. Association for Computational Linguistics, pages 1003 1011. http://aclweb.org/anthology/P09-1113.

Huu Thien Nguyen and Ralph Grishman. 2015. Event detection and domain adaptation with convolutional neural networks. In Proceedings of the 53rd Annual Meeting of the Association for Computational Linguistics and the 7th International Joint Conference on Natural Language Processing. Association for Computational Linguistics, pages 365-371. https://doi.org/10.3115/v1/P15-2060.

Thien Huu Nguyen, Kyunghyun Cho, and Ralph Grishman. 2016. Joint event extraction via recurrent neural networks. In Proceedings of the 2016 Conference of the North American Chapter of the Association for Computational Linguistics: Human Language Technologies. Association for Computational Linguistics, pages 300-309. http://www.aclweb.org/anthology/N16-1034.

Kevin Reschke, Martin Jankowiak, Mihai Surdeanu, Christopher D Manning, and Daniel Jurafsky. 
2014. Event extraction using distant supervision. In Proceedings of the Ninth International Conference on Language Resources and Evaluation. pages 4527-4531. http://www.lrecconf.org/proceedings/lrec2014/pdf/1127_Paper.pdf.

Mihai Surdeanu, Julie Tibshirani, Ramesh Nallapati, and D. Christopher Manning. 2012. Multi-instance multi-label learning for relation extraction. In Proceedings of the 2012 Joint Conference on Empirical Methods in Natural Language Processing and Computational Natural Language Learning. Association for Computational Linguistics, pages 455-465. http://aclweb.org/anthology/D12-1042.

Joseph Turian, Lev-Arie Ratinov, and Yoshua Bengio. 2010. Word representations: A simple and general method for semi-supervised learning. In Proceedings of the 48th Annual Meeting of the Association for Computational Linguistics. Association for Computational Linguistics, pages 384-394. http://aclweb.org/anthology/P10-1040.

Linlin Wang, Zhu Cao, Gerard de Melo, and Zhiyuan Liu. 2016. Relation classification via multi-level attention cnns. In Proceedings of the 54th Annual Meeting of the Association for Computational Linguistics. Association for Computational Linguistics, pages 1298-1307. http://www.aclweb.org/anthology/P16-1123.

Matthew D Zeiler. 2012. Adadelta: An adaptive learning rate method. arXiv preprint arXiv:1212.5701 https://arxiv.org/pdf/1212.5701.

Daojian Zeng, Kang Liu, Yubo Chen, and Jun Zhao. 2015. Distant supervision for relation extraction via piecewise convolutional neural networks. In Proceedings of the 2015 Conference on Empirical Methods in Natural Language Processing. Association for Computational Linguistics, pages 1753-1762. https://doi.org/10.18653/v1/D15-1203. 